\title{
Modeling of a geothermal and thermal solar process *
}

\author{
Julien Nou, Adama Traoré, Thierry Talbert, Monique Polit* \\ * Laboratoire PROMES (UPR CNRS 8521), \\ Universite de Perpignan Via Domitia, \\ 52 avenue Paul Alduy, 66860 Perpignan, France. \\ E-mail addresses: \{julien.nou, traore, talbert, polit\}@univ-perp.fr
}

\begin{abstract}
This paper is based on a project combining solar and geothermal energies used for heating an individual home located in a mountainous region of southern France (Saint-Pierre Dels Forcats town). A solar field consists of 10 thermal solar collectors, with a total area of $21.25 \mathrm{~m}^{2}$ which recharge thermally a borehole of $100 \mathrm{~m}$ of depth. A heat pump extracts the heat energy to restitute it to the home with a heating floor of $100 \mathrm{~m}^{2}$. The long-term objective is to develop a predictive control on the global system to realize financial and energy savings. In order to do this, a modeling of all the subsystems has to be done. This paper focuses of two of these sub-systems, the solar field and the heat pump. Depending on the level of knowledge of each subsystem, a physical model (thermal solar collectors) or a artificial intelligence methodology (heat pump) is used.
\end{abstract}

Keywords: Modeling; Renewable energy; Neuro-fuzzy; Thermal solar collectors; Heat pump; Borehole.

\section{INTRODUCTION}

On December 11, 1997, the Kyoto Protocol was adopted, and entered into force on February 16, 2005. This international agreement is linked to the United Nations Framework Convention on Climate Change (Kyoto protocol [1997]). The major feature of the Kyoto Protocol is that it sets binding targets for 37 industrialized countries and the European community for reducing greenhouse gas (GHG) emissions. They amount to an average of five per cent against 1990 levels over the five-year period 2008-2012, McIntyre [2009]. Thus, after the well-known greenhouse effects on the climate change, the direct consequences on the health are now brought to light. That is why it is of paramount interest to reinforce the global effort to reduce the GHG emissions in all sectors of activity using fossil energies. A large part of human energy consumption and GHG emissions are due to the building sector. In France, it represents $46 \%$ of the global energy consumed and $25 \%$ of the GHG emissions, ADEME [2007]. In this context, improving the building energetic behavior, without reducing the comfort, goes obviously through the decreasing of the dependency to fossil energies, developing the resort to renewable energies. That is why PROMES laboratory (ELIAUS Team) and Dominguez Énergie, a french company specialist on geothermal energy in south of France, have introduced a coupling of geothermal heat

\footnotetext{
* The authors would like to acknowledge, for their financial supports, the Languedoc-Roussillon region with the HELIOSOL project, the ANR (Agence Nationale de la Recherche) with the monitoring and modeling project SOLARGEOTHERM, and the University of Perpignan Via Domitia for the BQR (Bonus Qualite Recherche) with the SiP project (System in Package). They also want to thank their industrial partner, the Dominguez Énergie company.
}

pumps with thermal solar collectors to study a possibility of a supervised storage of the thermal energy in the project called "GEOHELIOS". Geothermal energy is a good candidate for heating buildings due to its free and continuous heat supply. Nevertheless, extracting heat from a borehole, using a heat pump, cools the subsoil locally and reduces the performance of the process. A solution, put in this paper to the test, consists in using the thermal solar energy to warm the ground up and to store energy. Indeed, the need for storage is closely associated with the use of intermittent renewable energy, because solar energy produces greatly variable heat, not correlated in time with the energy consumed for heating a house. That is why an efficient means to store this thermal energy is needed. Solar energy is mainly produced between the middle day, when the house chiefly needs energy to be heated, during the winter period, early in the morning and during the evening. While the thermal storage over a few hours or a few days is already efficient with hot-water tanks, or thermal inertia in walls and foundations, inter-seasonal storage of energy in a geological reservoir has not been widely studied. Some similar studies have already been carried out (TrillatBerdal [2007] and Hepbasli [2007]), but the works were focused on a thermal approach while the final objective of the present project is developing an optimal controller with the aim of improving the functioning of the considered system.

This paper is organized as follow: Section 2 presents the hybrid process. Section 3 and 4 describes the two main subsystems of the process to product and extract the thermal energy: the thermal solar collectors and the heat pump. In these sections the associated models are described and the results are given. Finally, Section 5 concludes the paper. 


\section{PROCESS DESCRIPTION}

The process considered in this study started by Dominguez Énergie in 2006. It is composed of two $100 \mathrm{~m}$ of depth boreholes, one heat pump of $15 \mathrm{~kW}$ (thermal power) including $3.9 k W_{e}$ (electrical power), one individual house equipped with a heating floor, $21.25 \mathrm{~m}^{2}$ of thermal solar panels. The instrumentation and the monitoring system (Figure 1) were set up by PROMES laboratory on February 2008 and achieved on July 2009.

On this site, the subsoil is composed of granite rock with static water at about $15 \mathrm{~m}$ of depth. This is an important parameter, to take in consideration, because water circulation in the subsoil could involve the dispersion of thermal energy, compromising the required storage. As depicted on figure 1, the system contains two independent hydraulic circuits: (i) the solar circuit and (ii) the heat pump circuit. The first one is subdivided into two isolated parts. The first part connects the thermal solar panels to a heat exchanger. The second part is a circuit back/forth, also divided in two parts, one for each borehole. Two pumps are installed on the solar circuit and manage the fluid flow rate. The various variables presented on figure 1 will be presented and defined in the following section.

\section{THERMAL SOLAR COLLECTORS MODELING}

\subsection{Thermal solar collectors description}

Eight sensors are integrated in the solar circuit. They measure the solar irradiance $\left(I_{S P}\right)$ received by the thermal solar panels, the outdoor temperature $\left(T_{O U T}\right)$, the outlet temperature of the solar panels $\left(T_{S P(m) / H E}\right)$, the inlet temperature from the solar field measured at the input of the heat exchanger $\left(T_{S P / H E(m)}\right)$, the outlet temperature of the heat exchanger towards the solar panels $\left(T_{H E(m) / S P}\right)$, the inlet temperature of the heat exchanger which comes from the borehole $\left(T_{B O / H E}\right)$ and the outlet temperature of the heat exchanger towards the boreholes $\left(T_{H E / B O}\right)$. Flow rates have been calculated taking into account the power of the two pumps and their functioning time, using the measurement of the pumps electrical current $\left(I_{P T}\right)$. The choice of these measurements is a key-point to model the functioning of the thermal solar panels. Indeed, the direct measurement at the output of the solar field and at the back of the heat exchanger was chosen because this is the suitable configuration allowing a good control of the two pumps.

These pumps, (Pump 1 and Pump 2 - ref. Wilo, Star-RS $25 / 6130 \mathrm{~mm}$ ) are used to manage the thermal fluid flow in the solar hydraulic circuits. Thus, the thermal energy, produced by the solar collectors, can recharge the subsoil. The first pump manages the fluid flow between the solar panels and the heat exchanger and the second one between the heat exchanger and the borehole. The two pumps work together using a on-off control and they start when the difference between the inlet temperatures coming from the solar panel (output of the solar field, $T_{S P(m) / H E}$ ) and the borehole, $T_{H E / B O}$, is higher than $7^{\circ} \mathrm{C}$. They stop when this difference is lower than $4^{\circ} \mathrm{C}$. Thus the mass flow rate is defined considering the density and the volume flow rate as shown in the equation (1).

$$
\dot{m}_{H E / S P}=\rho \dot{V}_{H E / S P}
$$

\subsection{Physical model}

As presented in this section, all the physical and thermal characteristics of the different solar collectors components are known, Khalfallaoui [2008]. Thus for its modeling, a physical approach have been preferred. This aspect exposed is in the following section.

The thermal solar collectors modeling takes into account the geometrical characteristics, the thermal and physical properties of the thermal fluid (60\% water and $40 \%$ monopropylene glycol) and the various temperatures of the subsystem $\left(T_{O U T}, T_{S P(m) / H E}\right.$, and $\left.T_{H E(m) / S P}\right)$. These parameters and variables are listed in the table (1).

\begin{tabular}{|c|c|}
\hline$\rho$ & Fluid density $\left(\mathrm{kg} \cdot \mathrm{m}^{-3}\right)$ \\
\hline $\mathrm{V}$ & Volume of the solar field circuit $\left(\mathrm{m}^{3}\right)$ \\
\hline$C_{p}$ & Fluid specific heat capacity $\left(\mathrm{J} \cdot \mathrm{kg}^{-1} \cdot \mathrm{K}^{-1}\right)$ \\
\hline$S_{c}$ & Total area of the solar field $\left(\mathrm{m}^{2}\right)$ \\
\hline$\eta_{0}$ & Optical efficiency \\
\hline$\phi$ & Solar radiation $\left(W \cdot \mathrm{m}^{-2}\right)$ \\
\hline$U_{L}$ & Global exchange coefficient $\left(W \cdot \mathrm{m}^{-2} \cdot \mathrm{K}^{-1}\right)$ \\
\hline$T_{i}$ & Inlet temperature $(K)$ \\
\hline$T_{O}$ & Outlet temperature $(K)$ \\
\hline$T_{O U T}$ & Outdoor temperature $(K)$ \\
\hline
\end{tabular}

Table 1. Nomenclature of the solar field

Considering the input temperature expression

$$
T_{i}=T_{H E(m) / S P}
$$

and the ouput temperature expression

$$
T_{o}=T_{S P(m) / H E}
$$

the energy study results in (4).

$$
\begin{array}{r}
\rho V C_{P} \frac{\partial T_{o}}{\partial t}(t)=\dot{m} C_{p}\left(T_{i}(t)-T_{o}(t)\right)+S_{c} \eta_{0} \phi(t) \\
+U_{L} S_{c}\left(T_{O U T}(t)-T_{o}(t)\right)
\end{array}
$$

With a discretization, it is possible to determine $T_{o}(k+1)$ as shown in (5).

$$
\begin{array}{r}
T_{o}(k+1)=T_{o}(k)+\Delta t\left[\dot{m} C_{p}\left(T_{o}(k)-T_{i}(k)\right)\right. \\
\left.+S_{c} \eta_{0} \phi(k)+U_{L} S_{c}\left(T_{O U T}(k)-T_{o}(k)\right)\right] /\left(\rho V C_{p}\right)
\end{array}
$$

\subsection{Results and discussions}

The development of the thermal solar collectors is based on the global exchange coefficient $\left(U_{L}\right)$ identification. The parameters: $\rho$ and $C_{p}$ have been calculated considering the thermal fluid characteristics of the monopropylene glycol $40 \%, S_{c}, V$ and $\eta_{0}$ are known and $\dot{m}$ has been measured. The calculation of this parameter is based on the error at $\mathrm{k}$ (instant) $\epsilon(k)$ between the simulated outputs $y_{\text {sim }}(k)$ and the experimental outputs $y_{\text {sim }}(k)$.

$$
\epsilon(k)=y_{\text {exp }}(k)-y_{\text {sim }}(k)
$$

Minimizing the sum of the quadratic errors $e$ on the $N$ measurements. 


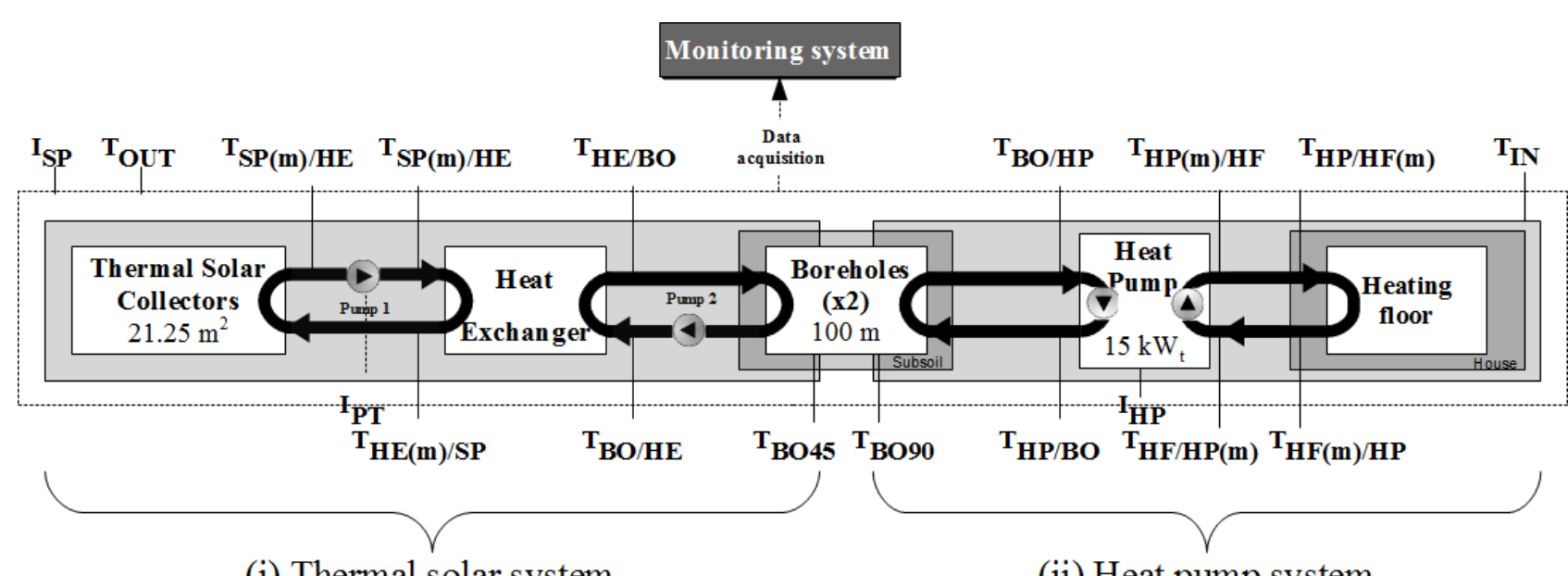

(i) Thermal solar system

(ii) Heat pump system

Figure 1. Synoptic of the instrumentation

$$
e=\sum_{k=1}^{N} \epsilon^{2}(k)
$$

In this framework, to minimize $e$ we use the algorithm of nonlinear least squares by the trust region Newton's method.

From then on, two criteria were considered in the optimization phase of the model parameters to avoid local minima. Indeed, the minimization of the quadratic errors sum is a first step to identify $U_{L}$. Consequently, it is necessary to validate or not this estimation by using others criteria. The first one is the goodness-of-FIT testing approach as presented in (8).

The calculation of the FIT factor makes it possible to evaluate the similarity between a vector of experimental data $V_{\text {exp }}$, and a vector of simulation $V_{\text {mod }}$. A value of $100 \%$ indicates a perfect similarity between the vectors being compared, whereas a value near $0 \%$ indicates that the vector models did not behave closest experimental vector than the average of this experimental vector $\left\langle V_{\text {exp }}\right\rangle$.

$$
F I T=100\left(1-\frac{\left\|V_{\text {mod }}-V_{\text {exp }}\right\|_{2}}{\left\|V_{\text {exp }}-\left\langle V_{\text {exp }}\right\rangle\right\|_{2}}\right)
$$

The second criterion of optimization was the mean relative error (MRE) as presented in (9). These factors traduce the error between estimations and real measurements.

$$
M R E=100\left\langle\left|V_{\text {mod }}-V_{\text {exp }}\right| /\left|\max \left(V_{\text {exp }}\right)-\min \left(V_{\text {exp }}\right)\right|\right\rangle
$$

The thermal solar collectors model was set up using data from October 2009 to March 2010.

Figure (2) shows an example of obtained results. On this figure, one can observe a good agreement between estimations and measurements. This observation can be confirmed since the FIT factor is evaluated to $85 \%$ when the ERM represents a very low value less than $3 \%$.

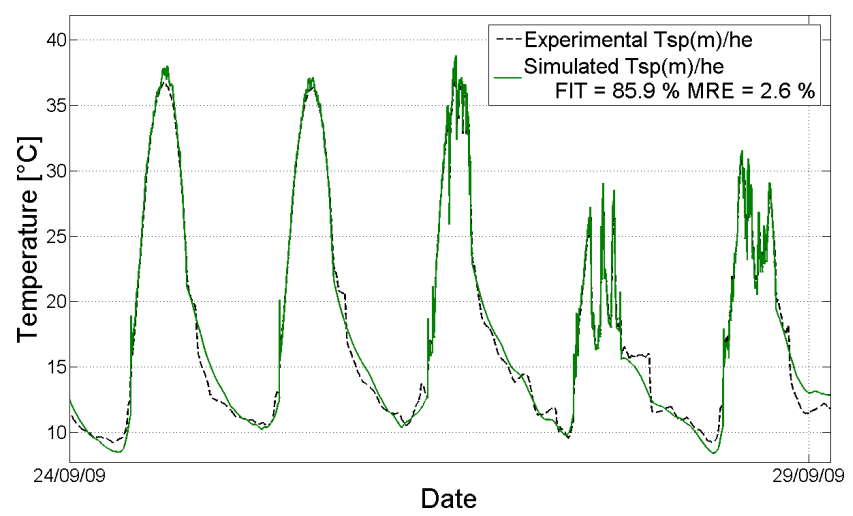

Figure 2. Simulation of the outlet solar panels temperature

\section{HEAT PUMP MODELING}

\subsection{Heat pump description}

The instrumentation of the heat pump circuit includes eight sensors measuring the temperature inside the house $\left(T_{I N}\right)$ and various temperatures of water flowing out, i.e. from the heat pump towards the heating floor (temperatures are measured near the heat pump $\left(T_{H P(m) / H F}\right)$ and near the heating floor $\left(T_{H P / H F(m)}\right)$, from the heating floor towards the heat pump (temperatures are measured near the heat pump $\left(T_{H F / H P(m)}\right)$ and near the heating floor $\left(T_{H F(m) / H P)}\right)$, from the heat pump towards the borehole $\left(T_{H P / B O}\right)$ and from the borehole towards the heat pump $\left(T_{B O / H P}\right)$. Moreover, the electrical current consumed by the heat pump $\left(I_{H P}\right)$ is measured and allows deducing when it works and also when fluid flows in the circuits. These measurements are useful for modeling the heat pump and observing in real time the heat power consumption of the house. As it can be noticed, a large set of measurements is realized on this process. Moreover, the human knowledge inform of expertise is available. To combine these two kinds of information, fuzzy logic (in particular neuro-fuzzy) approach have been preferred.

Before beginning to deal with the model architecture, it is important to explain that it could be possible to model the heat pump employing other methodologies as a physical model like the previous subsystem. However, 


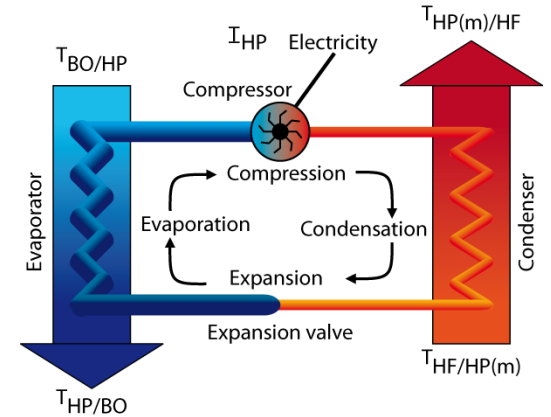

Figure 3. Heat pump functioning and measured temperatures

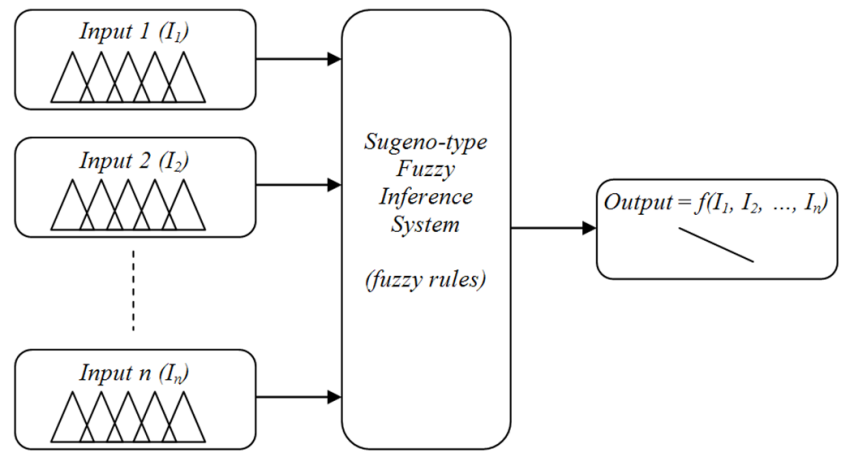

Figure 4. Anfis simplified framework

in this paper, the interest is to use a methodology based on the artificial intelligence and more precisely the neurofuzzy in order to allow us adding easily others inputs variables for future works based on the heat pump control. This tool is described in the following section.

\subsection{Adaptive neuro-fuzzy inference system (ANFIS)}

In the field of artificial intelligence, neural networks and fuzzy logic can be combined in neuro-fuzzy systems (NFS) in order to achieve both properties of readability and learning ability. Neuro-fuzzy systems synergizes the two techniques by combining the human-like reasoning style of fuzzy systems, through the use of fuzzy sets and a linguistic model based on "if-then" fuzzy rules, with both the learning (training) process and the connectionist structure of neural networks, Lin [1996], and Abraham [2005]. In the present work, an Adaptive Neuro-Fuzzy Inference System (ANFIS) has been used with the aim of modeling the heat pump.

ANFIS, as presented on figure 4, is the combination of an adaptive artificial neural network and a Sugenotype (or Takagi-Sugeno-Kang-type, Takagi [1985]). In this kind of fuzzy model, output membership functions are either linear or constant. Fuzzy Inference System (FIS) that maps (1) input characteristics to input membership functions, (2) input membership functions to fuzzy rules, (3) fuzzy rules to a set of output characteristics, (4) output characteristics to output membership functions, and (5) output membership functions to output characteristics, Jang [1993].

Let's remember that the main difference between Mamdani and Sugeno fuzzy inferences is that the Sugeno output membership functions are either linear or constant (Mam- dani [1974]). Membership functions are first fixed and somewhat arbitrary chosen. A hybrid learning algorithm, the combination of the least-squares and backpropagation gradient descent methods, is then used to both identify (adjust) the FIS membership functions parameters and emulate (approximate) a given input/output training data set through the design of fuzzy rules. As a consequence, the neuro-adaptive learning technique provides a method for the fuzzy modeling procedure to learn information about a data set, in order to compute the membership functions parameters that best allow the associated (and automatically-generated) fuzzy inference system to track the given input/output data. This allows the FIS to learn from the data it is trying to model, Jang [1993]. This modeling approach is similar to many system identification techniques and needs the training data to be fully representative of the features that the trained FIS intends to learn. Unfortunately, in some cases, data is incomplete, corrupted or collected through noisy measurements. That is why model validation is performed to check for model overfitting, using a checking data set, sufficiently distinct from the training data set so as not to render the validation process trivial and non-significant. In order to have an optimal choice of input parameters, a preliminary study was necessary. This is presented in the following section.

\subsection{Preliminary study}

A preliminary study on the input variables of the model was performed. The aim is to detect the best choice of input variables for the estimation of the outputs of the heat pump. After the analysis of these variables a good correlation between some of the measured variables were observed.

Based on our historical data but also using our expertise and our knowledge, it has been observed that the outdoor temperature and the temperature of the main room are needed. Table 2 shows the main possible combinations.

To achieve the model training (ANFIS) and to model the two outputs of the heat pump $\left(T_{H P / B O s i m}\right.$ and $\left.T_{H P / H F s i m}\right)$, it is imperative to use a set of data that is meaningful to the period of its use. In this framework, data collected during the complete heat period have been selected. Thus, measurement from the first half of each heating month (from October 2009 to March 2010) were considered for training. The second half being employed for the validation step.

Each combination takes into account the heat pump functioning state $(\mathrm{ON} / \mathrm{OFF})$. This variable is very important since it will be used in the control strategy to decide the actions on the heat pump power.

\begin{tabular}{|c|c|c|c|c|c|c|c|}
\hline & $\mathrm{A}$ & $\mathrm{B}$ & $\mathrm{C}$ & $\mathrm{D}$ & $\mathrm{E}$ & $\mathrm{F}$ & $\mathrm{G}$ \\
\hline$T_{B O / H P}$ & $\mathrm{x}$ & $\mathrm{x}$ & $\mathrm{x}$ & & $\mathrm{x}$ & $\mathrm{x}$ & \\
\hline$T_{H F / H P}$ & $\mathrm{x}$ & $\mathrm{x}$ & & $\mathrm{x}$ & $\mathrm{x}$ & $\mathrm{x}$ & $\mathrm{x}$ \\
\hline$T_{I N}$ & & & & & $\mathrm{x}$ & $\mathrm{x}$ & \\
\hline$T_{O U T}$ & & $\mathrm{x}$ & & & $\mathrm{x}$ & & $\mathrm{x}$ \\
\hline$I_{H P}$ & $\mathrm{x}$ & $\mathrm{x}$ & $\mathrm{x}$ & $\mathrm{x}$ & $\mathrm{x}$ & $\mathrm{x}$ & $\mathrm{x}$ \\
\hline
\end{tabular}

Table 2. Possible inputs combination

One could think that the case E (with all the available variables as inputs) is the best choice. In practice, two 
criteria are used for determining the best set of variables. The mean absolute error defined in (10) and the mean relative error (MRE).

$$
M A E=\left\langle\left|T_{\text {sim }}-T_{\text {exp }}\right|\right\rangle
$$

\subsection{Results and discussions}

Considering these two criteria, an optimized number of iterations and number (and shape) of membership was determined.

Considering the preliminary study, the best model is obtained in the case $\mathrm{B}$, where the following inputs are used: $T_{B O / H P}, T_{H F / H P}, T_{O U T}$ and $I_{H P}$. As shown in table 3 and 4 , the mean absolute error obtained is $0.76^{\circ} \mathrm{C}$ and the mean relative error is $4.8 \%$. The obtained model is a Sugeno-type fuzzy inference system (FIS). Two of the four inputs are subdivided into two Gaussian membership functions.

\begin{tabular}{|c|c|c|c|c|c|c|c|}
\hline \multicolumn{7}{|c|}{ Training } \\
\hline & $\mathrm{A}$ & $\mathrm{B}$ & $\mathrm{C}$ & $\mathrm{D}$ & $\mathrm{E}$ & $\mathrm{F}$ & $\mathrm{G}$ \\
\hline oct-mar & 0.50 & 0.48 & 1.1 & 1.0 & 0.33 & 0.35 & 0.71 \\
\hline \hline \multicolumn{8}{|c|}{ Validation } \\
\hline & $\mathrm{A}$ & $\mathrm{B}$ & $\mathrm{C}$ & $\mathrm{D}$ & $\mathrm{E}$ & $\mathrm{F}$ & $\mathrm{G}$ \\
\hline oct & 0.46 & 0.37 & 0.61 & 1.2 & 0.19 & 0.24 & 0.82 \\
\hline nov & 0.57 & 0.54 & 1.3 & 0.83 & 0.51 & 0.55 & 0.52 \\
\hline dec & 0.59 & 0.59 & 0.84 & 1.4 & 0.49 & 0.49 & 1.0 \\
\hline jan & 0.71 & 0.76 & 1.4 & 1.6 & 0.80 & 0.72 & 0.83 \\
\hline feb & 1.1 & 1.2 & 1.6 & 1.8 & 2.1 & 2.0 & 1.0 \\
\hline mar & 1.1 & 1.1 & 2.2 & 2.0 & 1.2 & 1.3 & 1.0 \\
\hline mean & 0.76 & 0.76 & 1.33 & 1.47 & 0.88 & 0.88 & 0.86 \\
\hline
\end{tabular}

Table 3. Absolute error on $T_{H P / H F s i m}$

\begin{tabular}{|c|c|c|c|c|c|c|c|}
\hline \multicolumn{7}{|c|}{ Training } \\
\hline & $\mathrm{A}$ & $\mathrm{B}$ & $\mathrm{C}$ & $\mathrm{D}$ & $\mathrm{E}$ & $\mathrm{F}$ & $\mathrm{G}$ \\
\hline oct-mar & 2.2 & 2.1 & 5.1 & 4.6 & 1.5 & 1.6 & 3.2 \\
\hline \hline \multicolumn{8}{|c|}{ Validation } \\
\hline & $\mathrm{A}$ & $\mathrm{B}$ & $\mathrm{C}$ & $\mathrm{D}$ & $\mathrm{E}$ & $\mathrm{F}$ & $\mathrm{G}$ \\
\hline oct & 3.8 & 3.1 & 5.1 & 10 & 1.5 & 2.0 & 6.8 \\
\hline nov & 3.4 & 3.2 & 7.9 & 5.0 & 3.0 & 3.25 & 3.1 \\
\hline dec & 3.9 & 3.9 & 5.6 & 9.1 & 3.3 & 3.22 & 6.9 \\
\hline jan & 4.7 & 5.1 & 9.2 & 10 & 5.3 & 4.75 & 5.5 \\
\hline feb & 7.2 & 7.5 & 9.9 & 11 & 13 & 12.5 & 6.5 \\
\hline mar & 6.1 & 6.0 & 12 & 11 & 6.5 & 7.11 & 5.8 \\
\hline mean & 4.9 & 4.8 & 8.28 & 9.35 & 5.43 & 5.47 & 5.77 \\
\hline
\end{tabular}

Table 4 . Mean relative error on $T_{H P / H F s i m}$

Figures 5 and 6 show that the simulation curves follow the experimental data, both for modeling $T_{H P / H F s i m}$ and $T_{\text {HP/BOsim. }}$. However, during times when temperatures values are minimum or maximum, the correlation between experimental and simulated values is greatly reduced. In fact, the main reason for this problem is the data acquisition frequency. Indeed, the acquisition system will measure the heat pump current $I_{H P}$ every minute. Thus, during this minute, the heat pump state can be $\mathrm{ON}$ or OFF.

\section{CONCLUSION}

The building sector being one of the largest sectors of energy consumption in Europe, and consequently one of

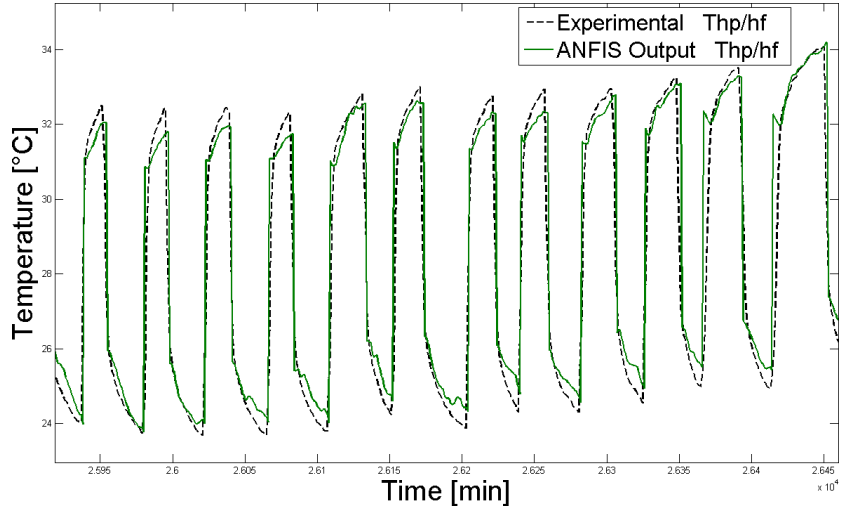

Figure 5. ANFIS output validation $T_{H P / H F}$

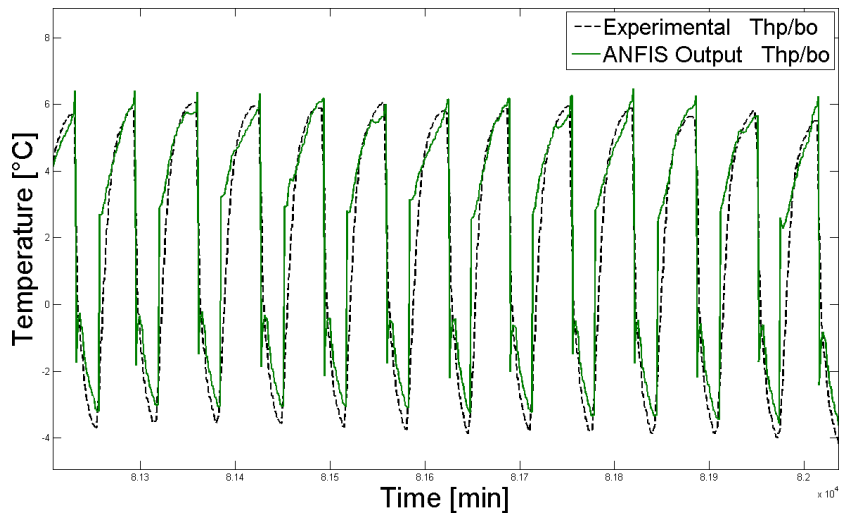

Figure 6. ANFIS output validation $T_{H P / B O}$

the major causes of greenhouse gas emissions, renewable energy is more and more an attractive alternative. In this framework the work presented in this paper is part of a general project of a hybrid installation energy management. The considered installation, using both solar and geothermal energies, is in Saint-Pierre Dels Forcats (south of France). For the modeling of this system, a subdivision into five subsystems have been realized. Particular attention was paid to the modeling of the thermal solar collectors and the heat pump because they represent the most important elements of the thermal production and extraction in the system. Associating real-time measurements, physical and expert knowledge, two models have been developed. The model proposed for the thermal solar collectors was a physical one, based on the energy study in the subsystem. The obtain results were satisfying since the FIT is about $86 \%$ and the MRE less than $3 \%$. The heat pump model was set up using artificial intelligence techniques namely, ANFIS algorithms. Considering the advantages of mixing various kinds of information, this approach provided very useful and interesting results in the estimation during the heat pump modeling since the validation was realized with a MRE of less than $5 \%$.

Analysing the obtained results, the two models presented can be validated and the more immediate continuation of this work is the modeling of the other subsystems. In this field research work is in hand. Will then follows the general model of the installation and the development of management strategies (control and supervision) in order to optimize the process performances improving the heat pump coefficient of performance (COP). 


\section{ACKNOWLEDGEMENTS}

Supported by the Languedoc-Roussillon region with the HELIOSOL project, the ANR (Agence Nationale de la Recherche) with the monitoring and modeling project SOLARGEOTHERM, the University of Perpignan Via Domitia with the SiP project (System in Package), and the industrial partner, the Dominguez Énergie company.

\section{REFERENCES}

United Nations Framework. Kyoto protocol to the united nations framework convention on climate change. Conference Kyoto-Japon, December 1997, united nations framework, 1998, 24 p., Cote : POL-005.

McIntyre S. Endangerment and Cause or Contribute Findings for Greenhouse Gases under the Clean Air Act. Docket ID No. EPA-HQ-OAR-2009-0171. Environmental Protection Agency, United States, 2009.

ADEME Les chiffres clés du bâtiment en 2006. Données et références, France, 2007.

Trillat-Berdal V., Souyri B., and Achard G. Coupling of geothermal heat pumps with thermal solar collectors. Applied Thermal Engineering. 27 (2007) 1750-5.

Hepbasli A. Exergetic modeling and assessment of solar assisted domestic hot water tank integrated groundsource heat pump systems for residences. Energy and Buildings. 39 (2007) 1211-7.

Khalfallaoui S., Seguin D., and Abdelghani-Idrissi A. Étude d'un système solaire thermique: Effet de l'orientation des panneaux solaires. XIV Colloque National de la Recherche dans les IUT, (2008).

Lin C.T., and Lee C. S. G. Neural Fuzzy Systems: A Neuro-Fuzzy Synergism to Intelligent Systems. Upper Saddle River, NJ: Prentice Hall, 1996.

Abraham A. Adaptation of Fuzzy Inference System Using Neural Learning, Fuzzy System Engineering: Theory and Practice. Nadia Nedjah et al. (Eds.), Studies in Fuzziness and Soft Computing, Springer Verlag Germany, Chapter 3, pages 53-83, 2005.

Jang J.S.R. ANFIS: Adaptive-Network-Based Fuzzy Inference System. IEEE Transactions on Systems, Man, and Cybernetics, Vol. 23, ${ }^{\circ}$ 3, 1993.

Takagi T., and Sugeno M. Fuzzy identification of systems and its applications to modeling and control. IEEE Transactions on Systems, Man, and Cybernetics, vol. 15, $\mathrm{n}^{\circ} 1$, p. 116-132, 1985.

Mamdani E.H. Application of fuzzy algorithms for simple dynamic plant. Proceedings IEEE, vol. 121, ${ }^{\circ} 12$, p.1585 - 1588, 1974. 